\title{
P04-11-11 Poster session
}

\section{Changes in glucocorticoid receptor- $\alpha$ profile in human placentae from full term and early term births}

\author{
Roselyn B Rose'meyer, Svetlana Bivol, Olivia J Holland, Suzzanne J Owen \\ Medical Sciences, Griffith University, Australia
}

Background; Glucocorticoids (GCs) play a pivotal role in a variety of physiological processes, including human reproduction, with cortisol levels rising towards term to facilitate fetal growth and parturition. Research suggests that GCs may regulate the length of gestation, with human male and female foetuses responding differentially when exposed to high maternal cortisol concentrations. Glucocorticoid receptors (GRs), through which GCs mediate their molecular actions, display a remarkable diversity as a result of alternative splicing and alternative translation initiation.

Methods; Since GCs may control the length of gestation via their cognate GRs, we examined glucocorticoid receptor patterns in early-term (37-38 weeks) and full term (39-40 weeks) placentae using Western blot techniques.

Results; The isoforms detected in both placental cytoplasmic and nuclear fractions were as follows: GR $\alpha-\mathrm{A}$, GR $\alpha-\mathrm{C}$, $\mathrm{GR} \alpha-\mathrm{D} 1, \mathrm{GR} \alpha \mathrm{D} 2 / \mathrm{D} 3, \mathrm{GR} \beta$ and unknown $68 \mathrm{kDa}$ and $38 \mathrm{kDa}$ proteins. GR $\alpha-\mathrm{A}$ and unknown $38 \mathrm{kDa}$ protein were higher in the nucleus than in the cytoplasm in all placentae independent of fetal sex or gestational age $(\mathrm{P}<0.05)$. There were significant sex differences in the nuclear GR profiles in relation to gestational age. While early-term female fetus placentae exhibited significantly higher nuclear expression of GR $\alpha-\mathrm{A}$, GR $\alpha-\mathrm{C}$, GR $\alpha-\mathrm{D} 1$, GR $\alpha$-D2/D3 , unknown 68 $\mathrm{kDa}$ and GR $\beta$ protein compared to the full-term female placentae $(\mathrm{P}<0.05)$, the placentae of early-term male fetuses had higher expression of nuclear GR $\alpha-\mathrm{A}$ and unknown $38 \mathrm{kDa}$ protein than the placentae of full-term males $(\mathrm{P}<0.05)$. Conclusions; Overall the results indicate a complicated interaction between multiple GR isoforms in regulating placental development with GR expression being dependent on fetal sex, gestational age and GC levels. A better understanding of how GCs induce alterations in the placental structure and function and affect in utero fetal development may improve the pregnancy outcomes for early term babies. 